Antanas Fursenko - Arturas Kilikevicius - Kristina Kilikeviciene - Jonas Skeivalas -Albinas Kasparaitis Jonas Matijosius - Dariusz Wieckowski

\title{
ANALYSIS OF DYNAMIC PARAMETERS OF THE SYSTEM OF RASTER FORMATION AND CONTROL
}

The presented research work analyzes the sensing system, the main aim of which is a raster formation and controlling this process using the optical measuring equipment and high precision angle encoders. Optical measuring equipment are used for the raster position detection, meanwhile angle encoders for controlling the tape speed. The main parameter of raster formation process is fixed transportation speed, but there are difficulties to realize it, because there is imperfection of the device elements. The article analyzes the dispersion of vibration accelerations of the raster formation device and tape in the two directions (transverse and longitudinal) and presents an analysis of their parameters in application of the theory of covariance functions. The results of the measurements of vibration accelerations at the fixed points of the device constructions and the tape were recorded on a time scale in the form of digital arrays (matrices). Values of auto-covariance and inter-covariance functions of digital arrays of the vibration accelerations measurement data were calculated by changing the quantum interval in a time scale. The developed software Matlab 7 in operator package environment was used in the calculations.

Keywords: raster formation and controlling sensing system, vibration signal accelerations, dynamic parameters, covariance function, quantum interval

\section{Introduction}

Steel tape, on the surface of which certain symbols, such as rasters, special marks and the like are formed, are often used for metrological and technological needs, such as for measuring the displacement. Those symbols are formed by the movement of a steel tape and the beams of the source of light, which affects either the band surface or its special coatings.

The undesired deviations in the position and the shape of symbols formed in that way mainly depend on uncontrolled deviations of the said movement of the tape and the light beam from the set parameters.

One of the most important components of such deviations are vibrations of the band resulting from the sources of excitation of the tape transporting system mechanism and other internal and external vibrations. Uncontrolled changes in their position and the displacement speed, as well as the band deformation that have an adverse effect on the quality of the formed structures, occur as a result of their impact. It also affects the accuracy and reliability of the active control of the process carried out in real time. This effect on the system of defined parameters depends on vibration parameters - frequencies, amplitudes and other statistical characteristics. Knowing these parameters is important for designing the construction and optimizing the processes of formation the construction formation and of the structure of symbols.

The presented research examines the tape transport system consisting of electromechanical tape tractions and its fixed tensioning mechanisms as well as a tape tilt mechanism operating on sliding friction. This system is mounted on a massive granite base built on the foundation using passive vibration insulators. The research and data processing method and results of the experimental study of the layout system are proposed in the article. Experience of this article authors in construction, analysis and optimization of mechatronic precision devices is presented in papers [1-4].

The formation process takes place in the dynamic mode because both the steel tape and the laser raster formation head are continuously moving during the process.

In assessment of the significant points of the construction being examined, analysis of the vibrations dispersion of construction of the device and its tape point was carried out by application of the covariance function theory.

The Gaussian process emulator, with separable covariance function assumption of separability, imposes constraints on the emulator and may negatively affect its performance in some applications where separability may not hold. Zhang et al. [5] propose a multi-output Gaussian

\footnotetext{
Antanas Fursenko $^{1}$, Arturas Kilikevicius ${ }^{1}$, Kristina Kilikeviciene ${ }^{2}$, Jonas Skeivalas ${ }^{3}$, Albinas Kasparaitis ${ }^{1}$, Jonas Matijosius ${ }^{1}$, Dariusz Wieckowski ${ }^{4}, *$ ${ }^{1}$ Institute of Mechanical Science, Vilnius Gediminas Technical University, Lithuania ${ }^{2}$ Department of Mechanical and Material Engineering, Vilnius Gediminas Technical University, Lithuania ${ }^{3}$ Department of Geodesy and Cadastre, Vilnius Gediminas Technical University, Lithuania ${ }^{4}$ Lukasiewicz R\&D Network Automotive Industry Institute, Warsaw, Poland

*E-mail of corresponding author: d.wieckowski@pimot.eu
} 
process emulator with a non-separable auto-covariance function to avoid limitations of using the separable emulators.

Guella et al. [6] provide characterization theorems for high dimensional spheres, as well as for the Hilbert sphere.

Kithulgoda et al. [7] present a strategy for incremental maintenance of the Fourier spectrum to changes in concept that take place in data stream environments. Singh and Singh [8] generalized Chebyshev-Fourier moments (G-CHFMs) and generalized pseudo - Jacobi-Fourier moments (G-PJFMs) have been extended to represent color images using quaternion algebra.

Zhang et al. [9] used the spectral densities to calculate the average entropy of mixtures of random density matrices and show that the average entropy of the arithmetic-meanstate of $n$ qubit density matrices randomly chosen from the Hilbert-Schmidt ensemble was never decreasing with the number $\mathrm{n}$. Kurt and Eryigit [10] studied dynamics of the two-state system coupled to an environment with peaked spectral density.

Alotta et al. [11] provided a complete characterization of normal multivariate stochastic vector processes. Band et al. [12] focused in fractal Fourier analysis in which the graphs of the basic functions are Cantor sets, discontinuous at a countable dense set of points, yet have good approximation properties. Zhang et al. [13] simulated fractal signals that were generated under varying signal lengths and scaling exponents that indicate a range of physiologically conceivable fractal signals. Liu et al. [14] aimed to improve the computational accuracy of the fractal dimension of the profile curve.

Florindo and Bruno [15] proposed a novel technique for the numerical calculus of the fractal dimension of fractal objects, which can be represented as a closed contour. Xie et al. [16] proposed a layered online data reconciliation strategy based on a Gaussian mixture model, which is proposed for the complex industrial processes with multiple modes. Odry et al. [17] demonstrated the use of a special test bench that both enables simulations of various dynamic behaviors of wheeled robots and measures their real attitude angles along with the raw sensor data. Song el al. [18] proposed a structure-preserving bilateral texture filtering algorithm to smooth the texture while preserving salient structures.

Du et al. [19] proposed a correlation filter based visual tracking approach that integrates spatial-temporal adaptive feature weights into the DCF method to derive an efficient solution. Li et al. [20] proposed an algorithm for adaptive multiscale morphological filtering (AMMF), and its effect was evaluated by a simulated signal. Nathen et al. [21] presented an extension of a Large Eddy Simulation based approach in the framework of Lattice Boltzmann Methods.

During the research, an analysis of the raster formation device construction and dispersion of vibration accelerations of its tape points was carried out.

The tape transport system consists of electromechanical tape traction and its fixed tensile mechanisms, as well as a tape thrust mechanism operating on slip friction. The main parameter of the raster formation process is fixed transportation speed, but there are difficulties to realize it, because there is some imperfection of the device elements. This method may be used to produce a precision metrological scale on a stainless steel tape. The covariance function theory was used in analysis of the vibration parameters. Graphical expressions of the covariance functions show change of probabilistic inter-dependence of vibration parameters in the timeline. This degree of change of the vibration parameters spatial relations depends on technological and dynamic properties of the tape device being examined.

During the experiment, four sensors were laid out on the structural elements of the device and the fifth sensor was placed on the tape of the device. Vibration accelerations of all the five sensors were measured in the longitudinal and transverse directions of the tape movement.

\section{Simulation of vibration parameters}

The research presents an option for calculating the most reliable trend value of the vibrations vector applying the method of the least squares. It is assumed that the vibration vector trend is a discrete quantity with a fixed value. Use of the least squares method partially eliminates random vibration errors. When processing the large-scale measurement data, the least squares method also provides asymptotically effective values of the calculated parameters in the case where the measurement data distribution is not normal.

The array of measurements of vibration accelerations consists of 5 vectors $\varphi$ (columns). Each vector is understood as a random function with random measurement errors. The most reliable trend value $\tilde{\varphi}$ which is also called a weighted average, is calculated for each vector $\varphi$ in application of the least squares method. An assumption that the trend value of the vector changes according to the harmonic law, where the forecasted wave length corresponds to the length of the vibration accelerations vector, is used. The parameter equation of the individual vector value $\varphi_{i}$ reads:

$\varepsilon_{i}=\varphi_{i}-a_{i} \tilde{\varphi}$

where $\varepsilon_{i}$ is a random acceleration error, $\varphi_{i}$ is an acceleration value and $\tilde{\varphi}$ is an acceleration vector trend. Coefficient $a_{i}$ is expressed as:

$a_{i}=\cos \Delta_{i}$

where $\Delta_{i}=\Delta \cdot i, \Delta=2 \pi / n$ is the value of the unit of measure, $\operatorname{rad}, i=1,2, \ldots, n$.

The following is the equation (1) in the matrix form:

$\varepsilon=\varphi-A \tilde{\varphi}$, 
where $\varepsilon$ is a random error vector, $\varphi=\left(\varphi_{1}, \varphi_{2}, \ldots, \varphi_{n}\right)^{T}$ is a vibration accelerations vector, $A=\left(a_{1}, a_{2}, \ldots, a_{n}\right)^{T}$ is a matrix of coefficients of parameter equations $(n \times 1)$.

The most reliable trend value of the vibration accelerations vector $\varphi$ is calculated in application of the condition of the least squares method:

$$
\phi=\varepsilon^{T} P \varepsilon=\min ,
$$

where $P$ is the diagonal matrix of weights of vibration accelerations $\varphi_{i}(n \times n)$

Weights of individual acceleration values $\varphi_{i}$ are calculated according to formula

$$
p_{i}=\frac{\sigma_{0}^{2}}{\sigma_{\varphi_{i}}^{2}}
$$

where $\sigma_{0}$ is the standard deviation of the measurement result $\varphi_{0}$ the weight whereof is accepted as equal to one $p_{0}=1$. Thus, the $\sigma_{0}$ value is selected freely, because it has no impact on the calculation results. Value of the measurement result $\varphi_{0}$ is selected so that weights $p_{i}$ were close to one (to reduce the scope of calculations).

Out of t equation

$u_{i}=\ln \varphi_{i}$

the following is derived:

$$
\sigma_{\varphi_{i}}=\sigma_{u i} \varphi_{i}
$$

Equation (7) shows that $\sigma_{\varphi_{i}}$ value depends on value of the vibration accelerations $\varphi_{i}$. Thus, the acceleration of a higher value is of lower accuracy, because $\varphi_{i} \gg \sigma_{u i}$.

Using Equation (5), the following expression is obtained:

$$
p_{i}=\frac{\sigma_{0}^{2}}{\sigma_{u_{i}}^{2} \varphi_{i}^{2}}=\varphi_{i}^{-2} \cdot 10^{-7}
$$

where the accepted average value $\frac{\sigma_{0}^{2}}{\sigma_{u i}^{2}}=10^{-7}$

The extremity of the function (4) is determined having calculated its partial derivatives according to the parameter $\tilde{\varphi}$, equated it to zero and solved the equation received:

$$
\frac{\partial \Phi}{\partial \tilde{\varphi}}=2\left(\frac{\partial \varepsilon}{\partial \tilde{\varphi}}\right)^{T} P \cdot \varepsilon=0
$$

Then, the following is obtained:

$$
-A^{T} P \varepsilon=0
$$

and

$$
A^{T} P A \tilde{\varphi}-A^{T} P \varphi=0
$$

The solution is equal to:

$$
\tilde{\varphi}=\left(A^{T} P A\right)^{-1} A^{T} P \varphi=N^{-1} \omega
$$

where

$$
\begin{aligned}
& N=\left(A^{T} P A\right)^{-1}, \\
& \omega=A^{T} P \varphi .
\end{aligned}
$$

The accuracy of the parameter values calculated in application of the least squares method is assessed using their covariance matrix $K_{\tilde{\varphi}}$. Since the trend of cases of this task is a quantity (a scalar), the following may be written:

$K_{\tilde{\varphi}}^{\prime}=\sigma_{\tilde{\varphi}}^{\prime 2}=\sigma_{0}^{\prime 2} N^{-1}$,

where $\sigma_{0}^{\prime}$ is a value of the standard deviation $\sigma_{0}$. It is assessed according to the formula

$$
\sigma_{0}^{\prime 2}=\frac{1}{n-1} \varepsilon^{T} P \varepsilon
$$

\section{Object of the research and measurement results}

This article examines a raster formation device (Figure 1). The research work analyzes the tape transport system consisting of the electromechanical tape traction and its fixed tensile mechanisms, as well as a tape tilt mechanism operating on sliding friction. Vibrations in two directions (longitudinally and transversely) were measured at 4 points of the raster device (point 1 - fixed tension mechanism of the tape; point 2 - tape displacement measurement system; point 3- tilting node; point 4 - traction - tension system) and on the metal tape.

The block diagram of the metal scale production device presented in Figure 1 comprises the following: 1 - tape retractor mechanism; 2 - granite base; 3 - system measuring the tape displacement (point 2); 4 - tilting node (point 3); 5 - error monitoring node; 6 - traction - tension system (point 4); 7 - tape retractor mechanism; 8 - mechanism covering the tape in protective film; 9 - tape tension control and management system; 10 - fixed tension mechanism of the tape (point 1); 11 - optic system for turning laser beam microscopes with CCD camera; 12 - scanner; 13 - laser.

The articles analyzes vibration signals of significant points of the metal scale production device, when the device is in operating mode. Measurements were conducted in the two directions $\mathrm{X}$ and $\mathrm{Y}$ (Figure 1). Figure $2(\mathrm{Y}$ direction) and Figure 4 (X direction) illustrate results of acceleration of these measurement points and the spectral density graphs of these accelerations are respectively presented in Figures 3 and 5.

Analysis of results, illustrated in Figures 2-5, reveals that the highest level of vibrations was observed in the traction - tension system (Figure 1, point 4) when assessing the vibrations of the longitudinal direction Y (up to 32 $\mathrm{mm} / \mathrm{s}^{2}$ ); when assessing the vibrations of the transverse direction $\mathrm{X}$, accordingly, the highest level of vibrations was observed in the fixed tension mechanism of the tape (Figure 1 , point 1 ) and was about $190 \mathrm{~mm} / \mathrm{s}^{2}$ (the main components 


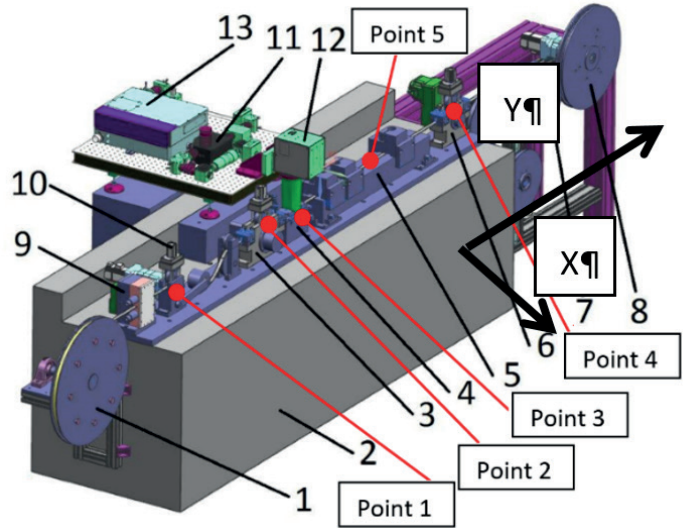

Figure 1 Block diagram of the metal scale production device with five measurement points

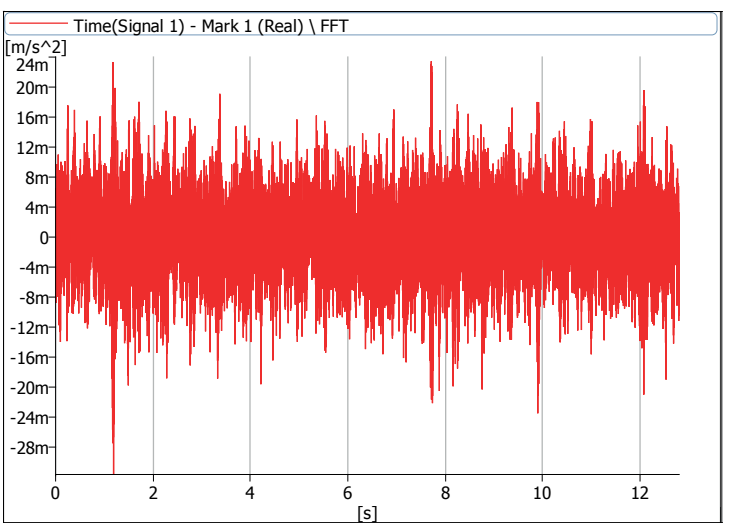

a)

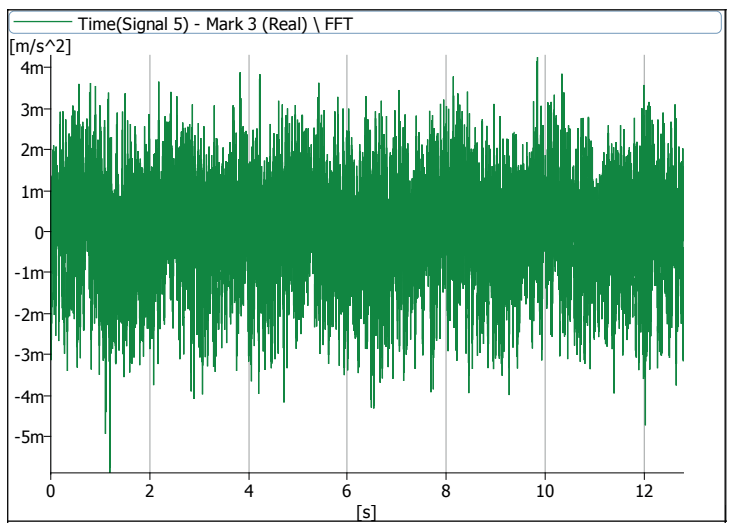

c)

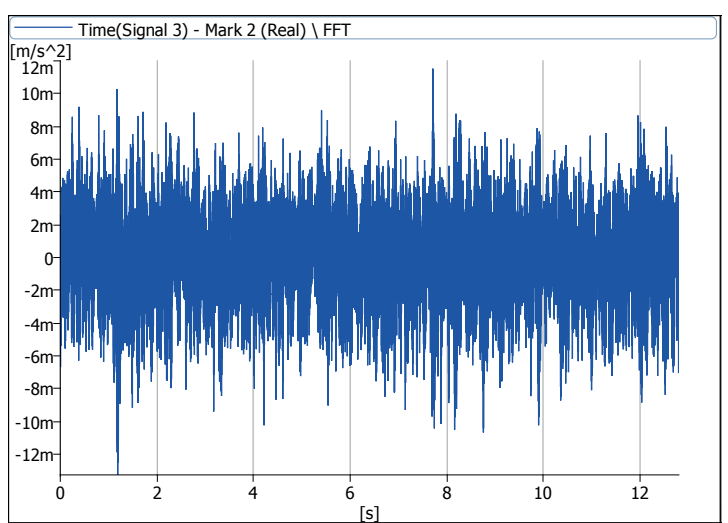

b)

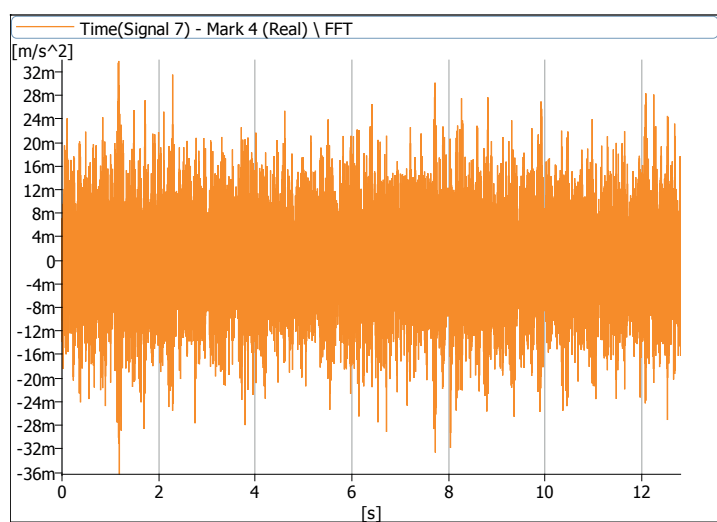

d)

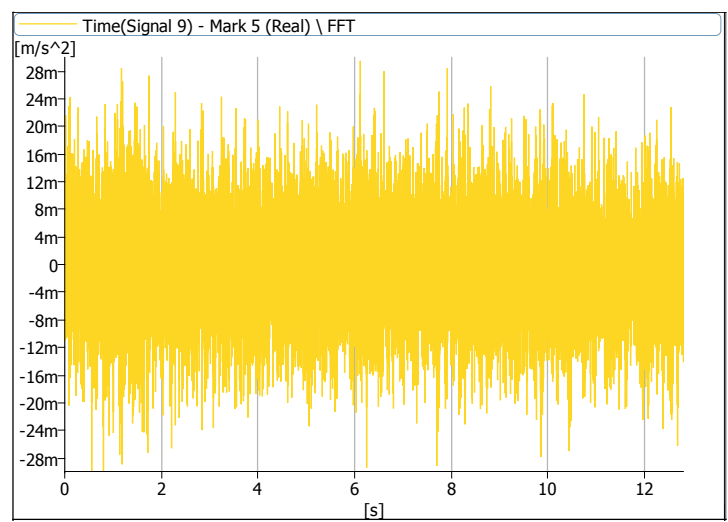

e)

Figure 2 Graphs of time signal for the $Y$ direction accelerations of measurement points (Figure 1): a) red - point 1; b) blue - point 2; c) green - point 3; d) orange - point 4; e) yellow - point 5 


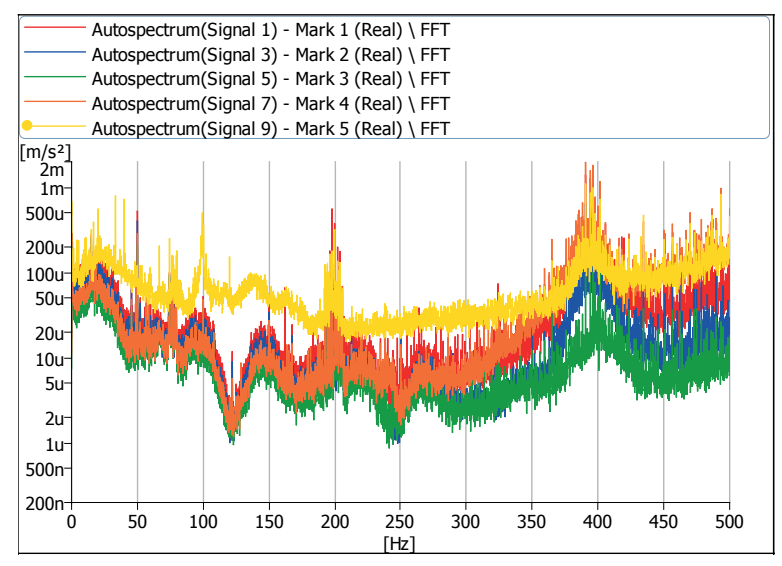

Figure 3 Graphs of spectral density for accelerations ( $Y$ direction in Figure 1)

of measurement points (red - point 1; blue - point 2; green - point 3; orange - point 4; yellow - point 5)

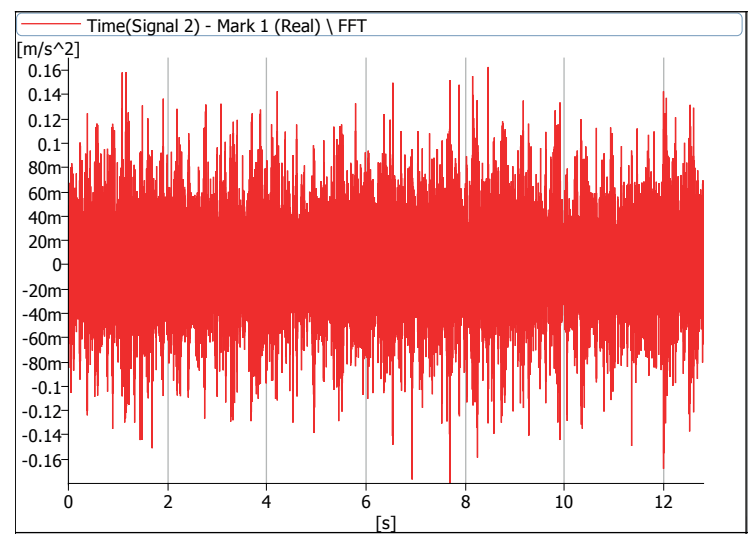

a)

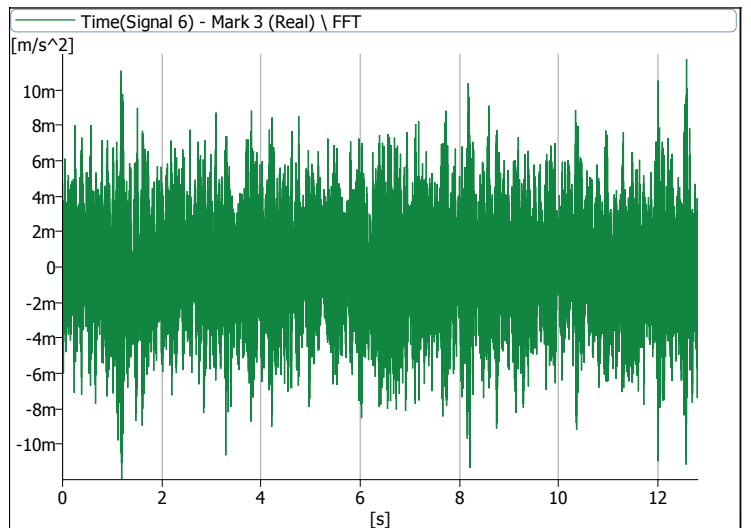

c)

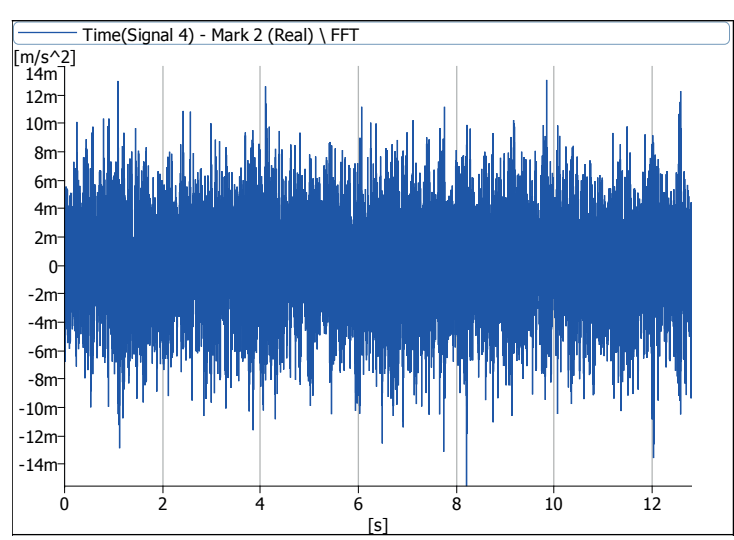

b)

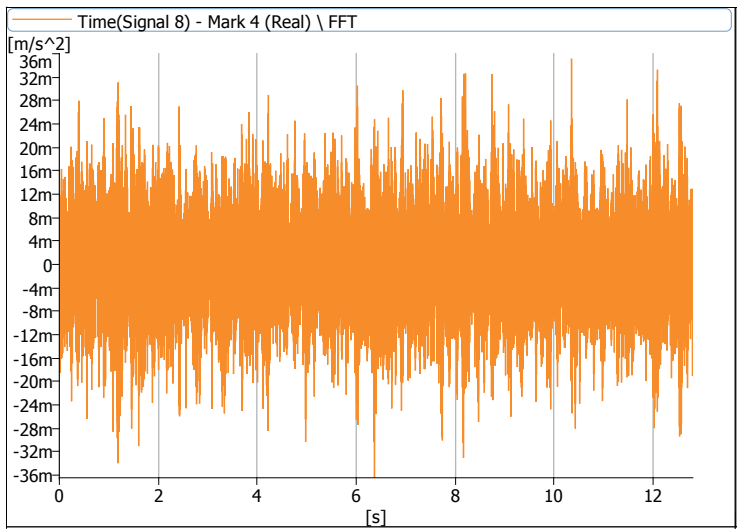

d)

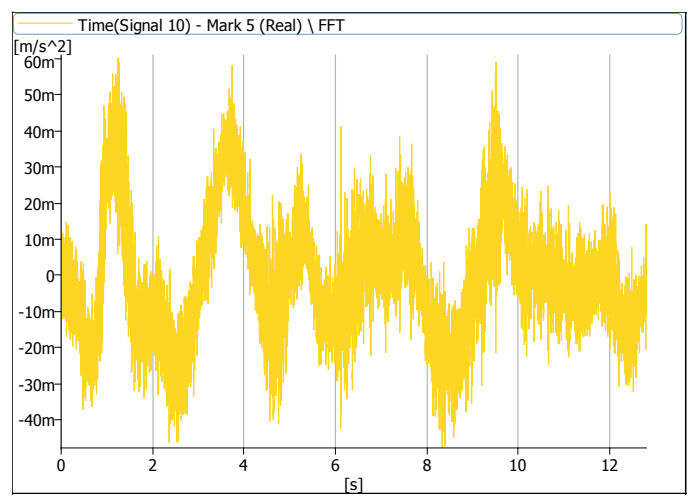

e)

Figure 4 Graphs of time signal for the $X$ direction accelerations of measurement points (Figure 1): a) red-point 1; b) blue - point 2; c) green - point 3; d) orange - point 4; e) yellow - point 5 


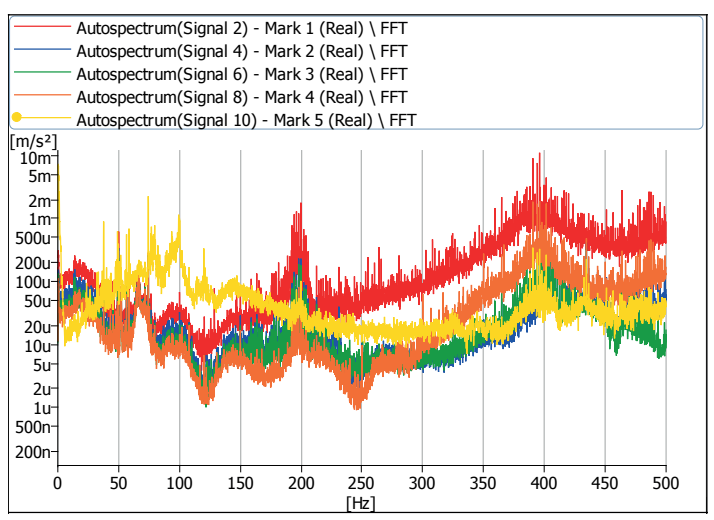

Figure 5 Graphs of spectral density for accelerations ( $X$ direction in Figure 1) of measurement points (red - point 1; blue - point 2; green - point 3; orange - point 4; yellow - point 5)

Table 1 Numbering of array vectors in the sequence order

\begin{tabular}{cc}
\hline Acceleration vector No. & Direction of the measurement of accelerations relative to the tape movement direction \\
\hline 1 & longitudinal \\
2 & transverse \\
3 & longitudinal \\
4 & transverse \\
5 & longitudinal \\
6 & transverse \\
7 & longitudinal \\
8 & transverse \\
9 & longitudinal \\
10 & transverse \\
\hline
\end{tabular}

were in the frequency range from 280 to $420 \mathrm{~Hz}$ from Figure 5). The lowest level of vibrations in the $Y$ and $X$ directions was in the tape tilting node (Figure 1, point 3), where the formation of rasters takes place and it was 6 (in the $\mathrm{Y}$ direction) and $12 \mathrm{~mm} / \mathrm{s}^{2}$ (in the $\mathrm{X}$ direction).

\section{Covariance model of vibration signal parameters} and the results of the analysis of the vibrations acceleration model

Guchhait and Banerjee [22] proposed a variant of constitutive equation error based material parameter estimation procedure for linear elastic plates, which was developed from partially measured free vibration signatures.

The conception of a stationary random function is the scientific ground of this theoretical model. Considering that, errors of measuring the bridge vibration parameters are random and of the same accurateness, ie. the average error $M \Delta=$ const $\rightarrow 0$ the dispersion $D \Delta=$ const . The covariance function of digital signals depends on the difference between the arguments only, ie. on the quantisation interval on the time scale [23].

The theoretical model was a subject of an assumption that errors of digital signals of vibrations are random and possibly systematic. The measurement data trend of the vector is eliminated in each vector of measurement arrays of vibration parameters. Vibration dispersion time interval is used as one of parameters. Tables 1 and 2 illustrate the schemes of the numbering of measurement arrays of the vibration accelerations vector. The quality of data of accelerations of all the five vectors was assessed using their accuracy indicator, a standard deviation. Standard deviation values are presented in Table 2.

Auto-covariance function of one data array or intercovariance function of the two arrays $K_{\varphi}(\tau)$ is expressed as [24-25]:

$K_{\varphi}(\tau)=M\left\{\varepsilon \overline{\bar{\varphi}}_{1}(u) \cdot \varepsilon \overline{\bar{\varphi}}_{2}(u+\tau)\right\}$

or

$K_{\varphi}(\tau)=\frac{1}{T-\tau} \int_{0}^{T-\tau} \varepsilon \varphi_{1}(u) \varepsilon \varphi_{2}(u+\tau) d u$

where $\varepsilon \varphi_{1}=\varphi_{1}-\overline{\varphi_{1}}, \quad \varepsilon \varphi_{2}=\varphi_{2}-\widetilde{\varphi_{2}}$ are centred measurement vectors of vibration parameters $\varphi$ with eliminated trend, $u$ is a vibrations parameter, $\tau=\tau \cdot \Delta$ is a variable quantum range, $k$ is a number of the measurement units, $\Delta$ is the value of the measurement unit, $T$ is time and $M$ is a symbol of the mean.

The covariance model of vibration signal parameters which authors used in this article is described in detail in papers [3, 23].

Arrays of the vibration accelerations measurements' data were obtained using 1-axis accelerometers 8344 and a 
Table 2 Accuracy indicators of the installation construction and vectors of vibration accelerations of the tape points

\begin{tabular}{|c|c|c|}
\hline $\begin{array}{l}\text { Name of } \\
\text { vectors }\end{array}$ & $\begin{array}{c}\text { Standard deviation value of single measurement result of vectors } \\
\text { Array }\end{array}$ & \\
\hline 1 & 0.006 & \\
\hline 2 & 0.041 & \\
\hline 3 & 0.003 & \\
\hline 4 & 0.003 & \\
\hline 5 & 0.001 & \\
\hline 6 & 0.003 & \\
\hline 7 & 0.008 & \\
\hline 8 & 0.017 & \\
\hline 9 & 0.008 & \\
\hline \multirow[t]{2}{*}{10} & 0.017 & \\
\hline & Vector parameter & Value $\mathrm{m} / \mathrm{s}^{2}$ \\
\hline 1 & Standard deviation value $\sigma^{\prime}{ }_{0}$ of the result with the weight of $p=1$ & 0.01 \\
\hline 6 & Standard deviation value $\sigma^{\prime}{ }_{0}$ of the result with the weight of $p=1$ & 0.01 \\
\hline 1 & Standard deviation value $\sigma_{v}^{\prime}$ of the weighted average of vectors & $1 \cdot 10^{-7}$ \\
\hline 6 & Standard deviation value $\sigma_{v}^{\prime}$ of the weighted average of vectors & $2 \cdot 10^{-7}$ \\
\hline
\end{tabular}

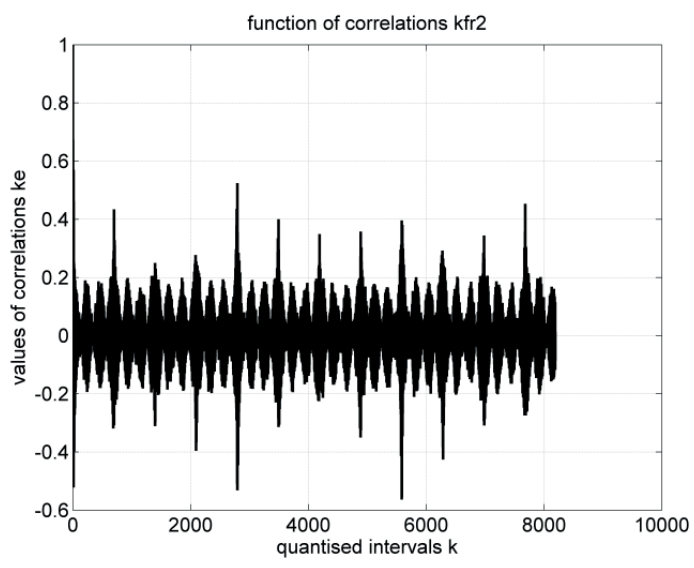

Figure 6 Simplified auto-covariance function of the 2 nd vibration accelerations vector of the array

tri-axial accelerometer 4506. Signals were recorded in the course of the time intervals $\tau_{\Delta}=0.00078 \mathrm{~s}$ and $12.8 \mathrm{~s}$. Values of $n=16386$ vibration signal accelerations were fit in each array vector.

Expression of each measurement vector is a random function due to random measurement errors when bringing its expression closer to the shape of a stationary function, trend components, which were calculated in application of the method of least squares, were eliminated.

Measurement data arrays were processed using the developed computer software in application of Matlab programme package operators.

Values of the quantised interval of simplified covariance functions range from 1 to $n / 2=8000$. The value $K_{\varphi}^{\prime}(\tau)$ of the simplified auto-covariance function $K_{\varphi}(\tau)$ was calculated for each vibrations vector and graphical expressions of 16 simplified auto-covariance functions were obtained.

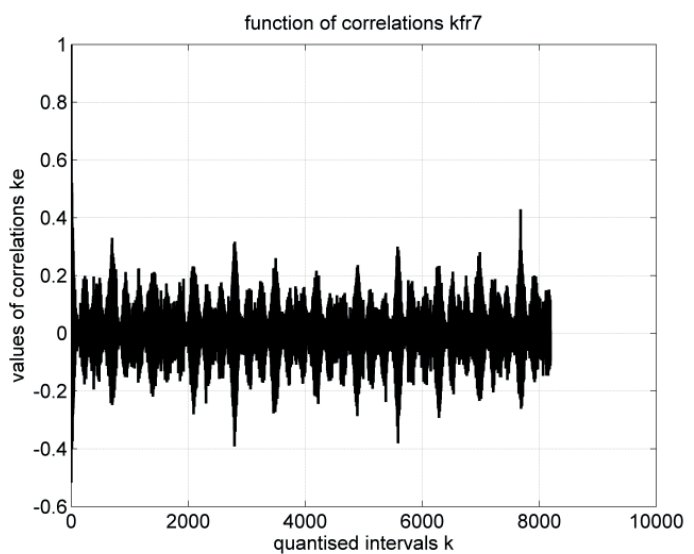

Figure 7 Simplified auto-covariance function of the 7th vibration accelerations vector of the array

Values of inter-normalized covariance functions $K_{\varphi}^{\prime}(\tau)$ s were also calculated based on 10 vibration array vectors and their graphical expressions were obtained according to their respective combinations.

Figures 6-17 illustrate more important graphical expressions.

Figure 18 presents the graphical image of the generalized (spatial) correlation matrix of the array of 8 vectors of vibration accelerations. Expression of the correlation matrix gets the block shape of 8 pyramids where the correlation coefficients values are shown in shades of the colour spectrum. The horizontal plane shows the colour projection of the pyramids.

Graphical expressions of the simplified auto-covariance and inter-covariance functions are of the two-tier shape. This is related to the fact that the structure of vibration signals is a composite function with vibrations of large amplitudes with long intervals and vibrations of small 


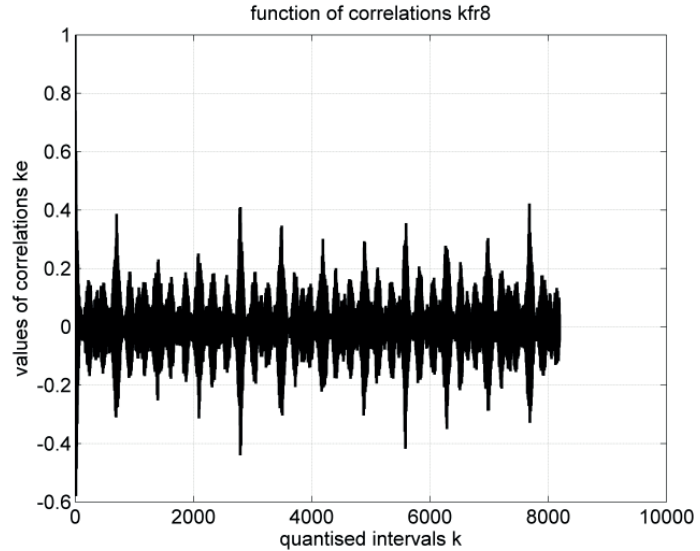

Figure 8 Simplified auto-covariance function of the 8th vibration accelerations vector of the array

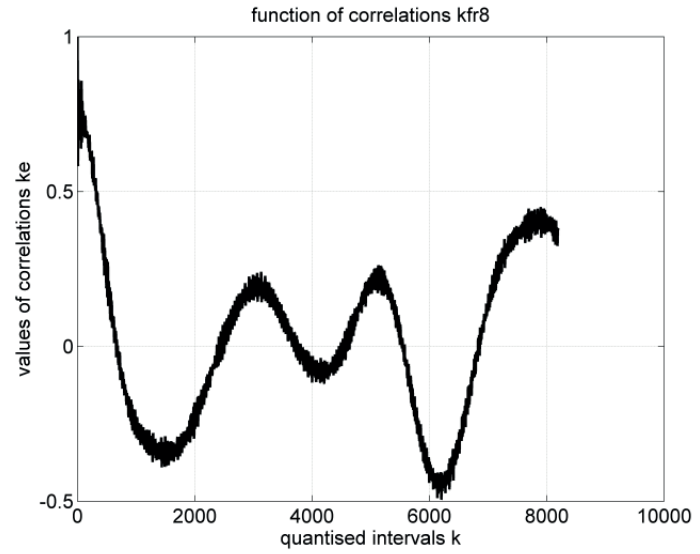

Figure 9 Simplified auto-covariance function of the 10(8) th vibration accelerations vector of the array

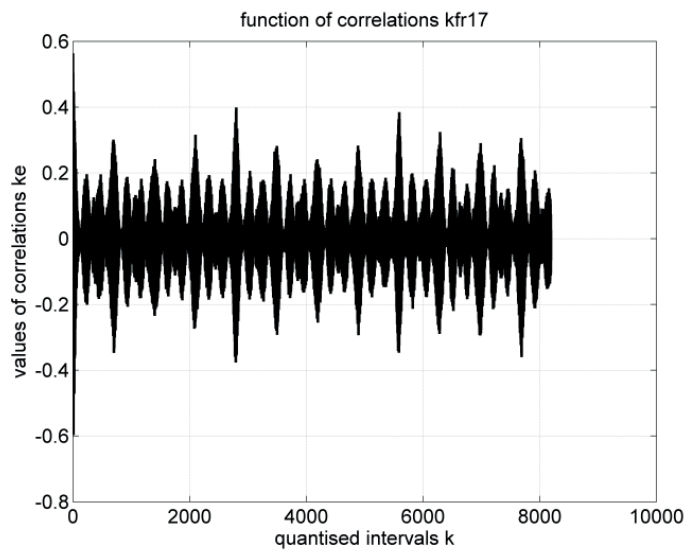

Figure 10 Simplified inter-covariance function of the 1st and 7th vibration accelerations vectors of the array

amplitudes. This determines a two-tier expression of the covariance functions.

The simplified auto-covariance functions of vectors of all accelerations of the arrays get the highest correlation coefficient values $r \rightarrow 1$ at values $\left(\tau_{k} \rightarrow 0 s\right)$ of the quantised interval and then decrease to $r \rightarrow 0$ at $k \rightarrow 10\left(\tau_{k} \rightarrow 0.0078 s\right)$ as a result of vibrations of small

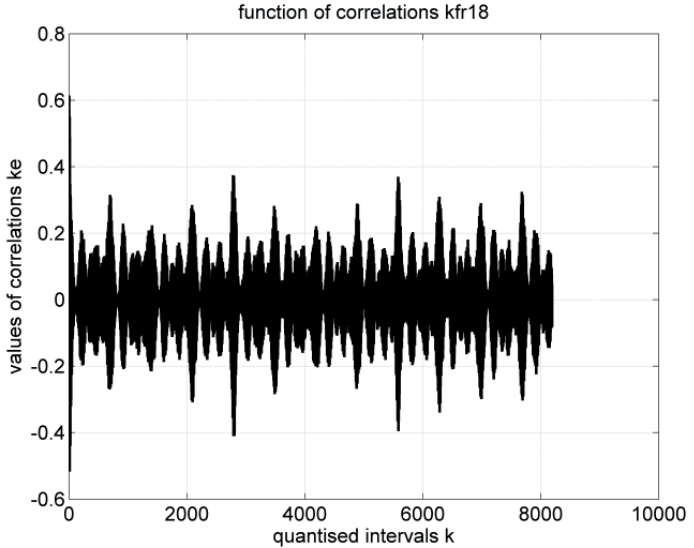

Figure 11 Simplified inter-covariance function of the 1st and 8th vibration accelerations vectors of the array

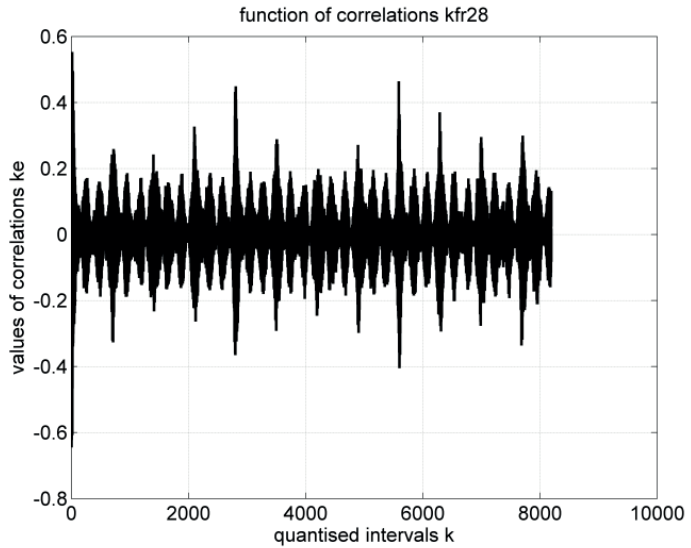

Figure 12 Simplified inter-covariance function of the 2nd and 8th vibration accelerations vectors of the array

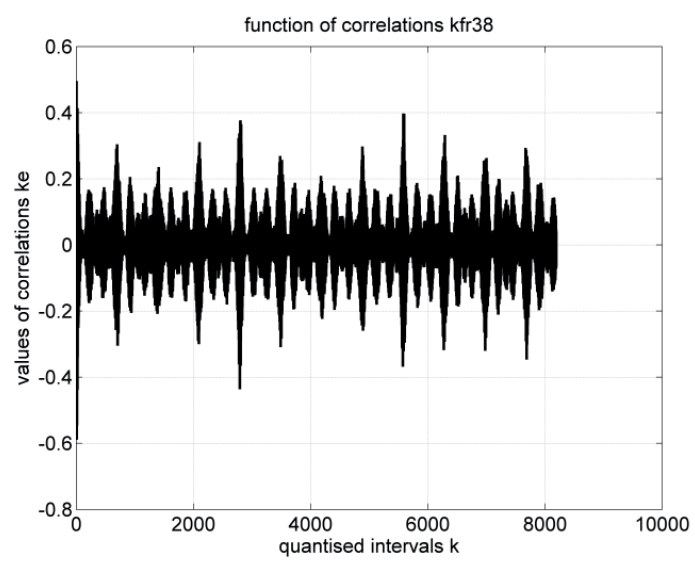

Figure 13 Simplified inter-covariance function of the 3rd and 8th vibration accelerations vectors of the array

amplitudes and at $k \rightarrow 1000\left(\tau_{k} \rightarrow 0.78 s\right)$ as a result of vibrations of large amplitudes. Expressions of simplified auto-covariance functions of acceleration vectors of the arrays are similar.

The $10^{\text {th }}$ simplified array auto-covariance function of the transverse vibrations accelerations vector of the tape has an expression that is close to the theoretical shape. 


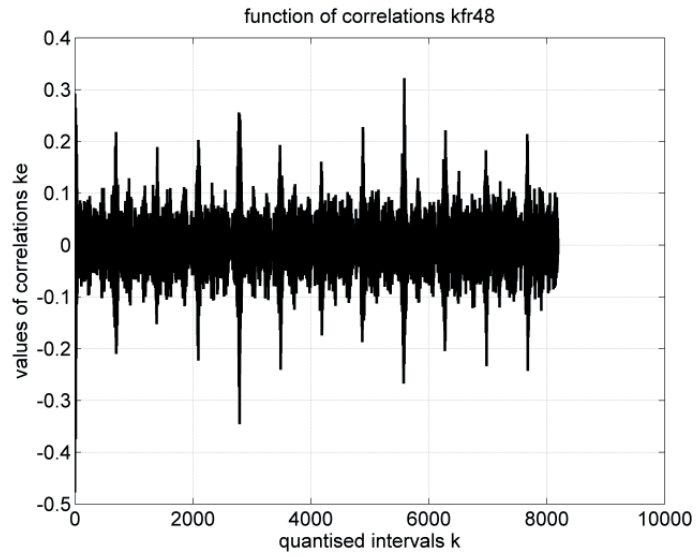

Figure 14 Simplified inter-covariance function of the 4 th and 8th vibration accelerations vectors of the array

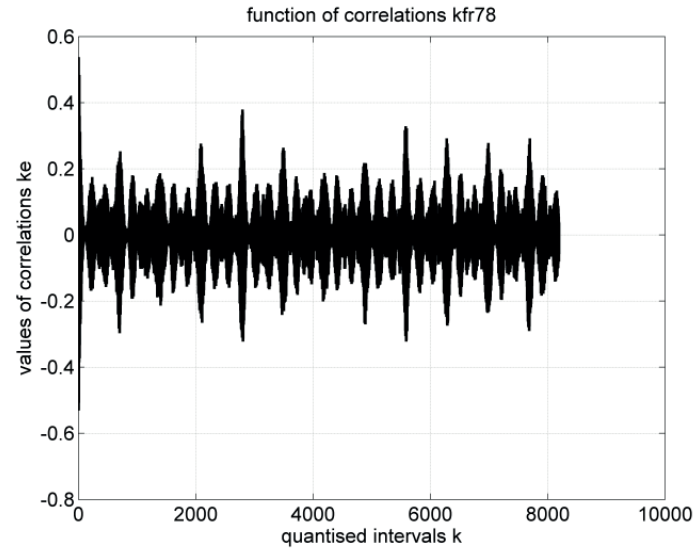

Figure 15 Simplified inter-covariance function of the rth and 8 th vibration accelerations vectors of the array

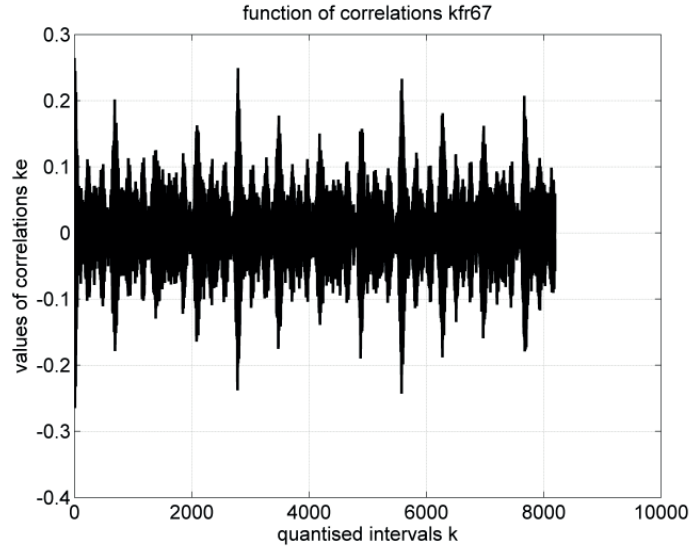

Figure 16 Simplified inter-covariance function of the 6th and $9(7)$ th vibration accelerations vectors of the array

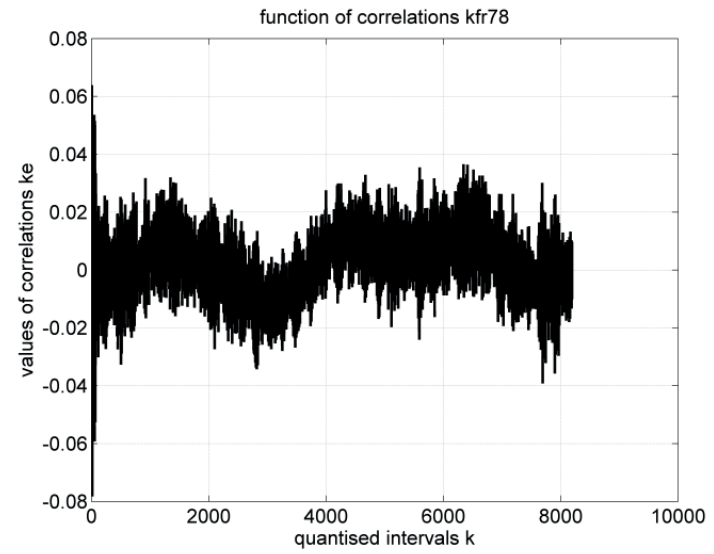

Figure 17 Simplified inter-covariance function of the $9(7)$ th and $10(8)$ th vibration accelerations vectors of the array

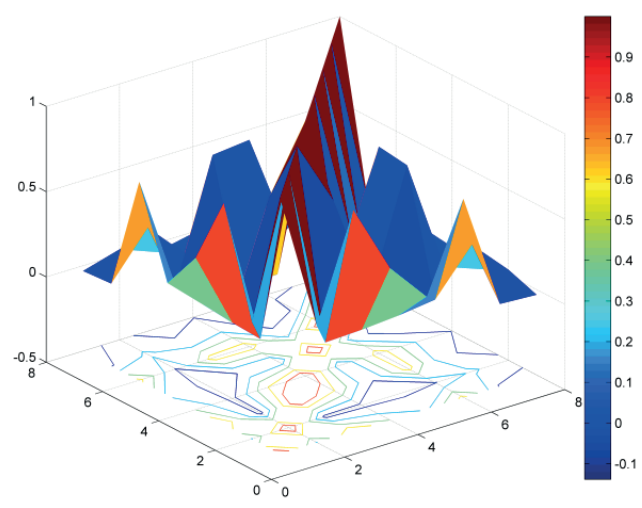

Figure 18 Graphical image of the generalized (spatial) correlation matrix of the 1st array of 8 vibration accelerations vectors

The simplified inter-covariance functions of the acceleration vectors of the array have average correlation coefficient values for the majority of pairs of vectors ranging in the 0.4-0.8 interval, which is illustrated by the data of inter-correlation of the $1^{\text {st }}$ acceleration vector with vectors of all the other 7 accelerations and between vectors 2 and 3, 2 and 7, 2 and 8, 3 and 6,3 and 7, 3 and 8, and 6 and 8 . Thus the correlation between the majority of accelerations vectors is significant enough, which shows potentially inter-related vibrations of nodes of the device construction.

\section{Conclusions}

Analysis of the dynamic parameters of the sensing system construction, the main aim of which is the raster formation and controlling this process, using the optical 
measuring equipment and high precision angle encoders, revealed that the highest level of vibrations of up to 32 $\mathrm{mm} / \mathrm{s}^{2}$ was in the traction - tension system, when assessing the longitudinal vibrations; when assessing vibrations in the transverse direction the highest level of up to $190 \mathrm{~mm} /$ $\mathrm{s}^{2}$ was formed in the fixed tension mechanism of the tape. Main components of the vibration acceleration amplitudes were in the frequency range from 280 to $420 \mathrm{~Hz}$.

The simplified auto-covariance and inter-covariance functions show the change of the probabilistic interdependence of vibration parameters of the raster formation tape device in the time scale. This degree of changes depends on the relation between vibrations of the structural nodes of the device being examined and the dynamic properties.

Graphical expressions of simplified auto-covariance and inter-covariance functions are two-tiered, which is related to the fact that the structure of vibration signals is a composite function that has vibrations of large amplitudes with long intervals and vibrations of relatively small amplitudes. Expressions of simplified auto-covariance functions of accelerations vectors of the arrays are similar.

The $10^{\text {th }}$ simplified auto-covariance function of the vector of transverse vibration accelerations of the tape has a different expression than other auto-covariance functions.

The simplified inter-covariance functions of accelerations vectors of the array have the average correlation coefficient values ranging within the 0.4-0.8 interval in the majority of pairs of vectors. Thus, the correlation between the majority of pairs of accelerations vectors is significant enough, which shows the possibly inter-related vibrations of construction nodes of the device.

\section{References}

[1] KILIKEVICIUS, A., KASPARAITIS, A. Dynamic research of multi-body mechanical systems of angle measurement. International Journal of Precision Engineering and Manufacturing [online]. 2017, 18(8), p. 1065-1073. ISSN 2234-7593, eISSN 2005-4602. Available from: https://doi.org/10.1007/s12541-017-0125-1

[2] SIAUDINYTE, L., KILIKEVICIUS, A., SABAITIS, D., GRATTAN, K. T. V. Modal analysis and experimental research into improved centering-leveling devices. Measurement [online]. 2016, 88, p. 9-17. ISSN 0263-2241. Available from: https://doi.org/10.1016/j.measurement.2016.01.044

[3] JUREVICIUS, M., SKEIVALAS, J., KILIKEVICIUS, A., TURLA, V. Vibrational analysis of length comparator. Measurement [online]. 2017, 103, p. 10-17. ISSN 0263-2241. Available from: https://doi.org/10.1016/j.measurement.2017.02.010

[4] KILIKEVICIUS, A. KASPARAITIS, A., LAZDINAS, R., KILIKEVICIENE, K. Kinematic and dynamic errors during the measurement of linear displacements using the angle transducers. Mechanics [online]. 2016, 22(3), p. 212-216. ISSN 1392-1207. Available from: https://doi.org/10.5755/j01.mech.22.3.14180

[5] ZHANG, B., KONOMI, B. A., SANG, H., KARAGIANNIS, G., LIN, G. Full scale multi-output Gaussian process emulator with nonseparable auto-covariance functions. Journal of computational physics [online]. 2015, 300, p. 623-642. ISSN 0021-9991. Available from: https://doi.org/10.1016/j.jcp.2015.08.006

[6] GUElla, J. C., MENEGATTO, V. A., PORCU, E. Strictly positive definite multivariate covariance functions on spheres. Journal of Multivariate Analysis [online]. 2018, 166, p. 150-159. ISSN 0047-259X. Available from: https://doi.org/10.1016/j.jmva.2018.03.001

[7] KITHUlGODA, C. I., PEARS, R., NAEEM, M. A. The incremental Fourier classifier: leveraging the discrete Fourier transform for classifying high speed data streams. Expert Systems with Applications [online]. 2018, 97, p. 1-17. ISSN 0957-4174. Available from: https://doi.org/10.1016/j.eswa.2017.12.023

[8] SINGH, C., SINGH, J. Quaternion generalized Chebyshev-Fourier and pseudo-Jacobi-Fourier moments for color object recognition. Optics and Laser Technology [online]. 2018, 106, p. 234-250. ISSN 0030-3992. Available from: https://doi.org/10.1016/j.optlastec.2018.03.033

[9] ZHANG, L., WANG, J., CHEN, Z. Spectral density of mixtures of random density matrices for qubits. Physics Letters A [online]. 2018, 382(23), p. 1516-1523. ISSN 0375-9601. Available from: https://doi.org/10.1016/j.physleta.2018.04.018

[10] KURT, A., ERYIGIT, R. Dynamics of a spin-boson model with structured spectral density. Physics Letters A [online]. 2018, 382(19), p. 1262-1267. ISSN 0375-9601. Available from: https://doi.org/10.1016/j.physleta.2018.03.021

[11] ALOTTA, G., DI PAOLA, M., PINNOLA, F. P. Cross-correlation and cross-power spectral density representation by complex spectral moments. International Journal of Non-Linear Mechanics [online]. 2017, 94, p. 20-27. ISSN 0020-7462. Available from: https://doi.org/10.1016/j.ijnonlinmec.2017.02.001

[12] BANDT, C., BARNSLEY, M. HEGLAND, M., VINCE, A. Old wine in fractal bottles I: Orthogonal expansions on self-referential spaces via fractal transformations. Chaos Solitons and Fractals [online]. 2016, 91, p. 478-489. ISSN 0960-0779. Available from: https://doi.org/10.1016/j.chaos.2016.07.007

[13] ZHANG, Z., VANSWEARINGEN, J., BRACH, J. S., PERERA, S., SEJDIC, E. Most suitable mother wavelet for the analysis of fractal properties of stride interval time series via the average wavelet coefficient method. Computers in Biology and Medicine [online]. 2017, 80, p. 175-184. ISSN 0010-4825. Available from: https://doi.org/10.1016/j. compbiomed.2016.11.009 
[14] LIU, Y., WANG, Y., CHEN, X., ZHANG, C., TAN, Y. Two-stage method for fractal dimension calculation of the mechanical equipment rough surface profile based on fractal theory. Chaos Solitons and Fractals [online]. 2017, 104, p. 495-502. ISSN 0960-0779. Available from: https://doi.org/10.1016/j.chaos.2017.09.012

[15] FLORINDO, J. B., BRUNO, O. M. Closed contour fractal dimension estimation by the Fourier transform. Chaos Solitons and Fractals [online]. 2011, 44(10), p. 851-861. ISSN 0960-0779. Available from: https://doi.org/10.1016/j. chaos.2011.07.008

[16] XIE, S., YANG, C., YUAN, X. WANG, X., XIE, Y. Layered online data reconciliation strategy with multiple modes for industrial processes. Control Engineering Practice [online]. 2018, 77, p. 63-72. ISSN 0967-0661. Available from: https://doi.org/10.1016/j.conengprac.2018.05.002

[17] ODRY, A., FULLER, R., RUDAS, I. J., ODRY, P. Kalman filter for mobile-robot attitude estimation: Novel optimized and adaptive solutions. Mechanical Systems and Signal Processing [online]. 2018, 110, p. 569-589. ISSN 0888-3270. Available from: https://doi.org/10.1016/j.ymssp.2018.03.053

[18] SONG, C., XIAO, C., LI, X., LI, J., SUI, H. Structure-preserving texture filtering for adaptive image smoothing. Journal of Visual Languages and Computing [online]. 2018, 45, p. 17-23. ISSN 1045-926X. Available from: https://doi.org/10.1016/j.jvlc.2018.02.002

[19] DU, F., LIU, P., ZHAO, W., TANG, X. Spatial-temporal adaptive feature weighted correlation filter for visual tracking. Signal Processing: Image Communication [online]. 2018, 67, p. 58-70. ISSN 0923-5965. Available from: https://doi.org/10.1016/j.image.2018.05.013

[20] LI, Y., ZUO, M. J., LIN, J., LIU, J. Fault detection method for railway wheel flat using an adaptive multiscale morphological filter. Mechanical Systems and Signal Processing [online]. 2017, 84, p. 642-658. ISSN 0888-3270, eISSN 1096-1216. Available from: https://doi.org/10.1016/j.ymssp.2016.07.009

[21] NATHEN, P., HAUSSMANN, M., KRAUSE, M. J., ADAMS, N. A. Adaptive filtering for the simulation of turbulent flows with lattice Boltzmann methods. Computers and Fluids [online]. 2018, 172, p. 510-523. ISSN 0045-7930. Available from: https://doi.org/10.1016/j.compfluid.2018.03.042

[22] GUCHHAIT, S., BANERJEE, B. Constitutive error based parameter estimation technique for plate structures using free vibration signatures. Journal of Sound and Vibration [online]. 2018, 419, p. 302-317. ISSN 0022-460X. Available from: https://doi.org/10.1016/j.jsv.2018.01.020

[23] SKEIVALAS, J., JUREVICIUS, M., KILIKEVICIUS, A., TURLA, V. An analysis of footbridge vibration parameters. Measurement [online]. 2015, 66, p. 222-228. ISSN 0263-2241. Available from: https://doi.org/10.1016/j. measurement.2015.02.034

[24] LI, Y., MULANI, S. B., SCOTT, K. M. L., KAPANIA, R. K., WU, S., FEI, Q. Non-stationary random vibration analysis of multi degree systems using auto-covariance orthogonal decomposition. Journal of Sound and Vibration [online]. 2016, 372, p. 147-167. ISSN 0022-460X. Available from: https://doi.org/10.1016/j.jsv.2016.02.018

[25] BAI, Z., WANG, C. A note on the limiting spectral distribution of a symmetrized auto-cross covariance matrix. Statistics and Probability Letters [online]. 2015, 96, p. 333-340. ISSN 0167-7152. Available from: https://doi.org/10.1016/j.spl.2014.10.002 